Conclusion Our findings are consistent with hypothesis that B cell response in RA synovium is probably antigen driven and oligoclonal.

The project was supported by grant VS96129 from Czech Ministry of Education, Youth and Sport in the Czech Republic.

\section{SLE - Clinical aspects}

\section{AB0071 LYMPHOCYTOTOXIC ANTIBODY (LCA) AMONG IRAQI SLE PATIENTS AND THEIR RELATIVES}

${ }^{1}$ MM Hasan, ${ }^{2} \mathrm{KM}$ Mousawy, ${ }^{2} \mathrm{~S}$ Salman, A Al-Wahab, ${ }^{2}$ AR Al-Shaikly. ${ }^{1}$ Faculty of Pharmacy

10.1136/annrheumdis-2001.116

${ }^{2}$ Faculty of Medicine, Baghdad University, Baghdad, Iraq

Background

Objectives To study the Lymphocytotoxic antibodies (LCA) in SLE patients and their first degree relatives compared to matching controls.

Methods Complement dependent microcytotoxicity test was used to assess the presence of LCA, their temperature dependence in addition to HLA specificity among 40 SLE patients, 184 of their first degree relatives in comparison to 100 subjects of a blood bank donor as a healthy control.

Results Lyphocytotoxic activity was manifested in $70 \%$ of patients, $33.36 \%$ of relatives compared to $80 \%$ of control group. While cold dependent antibodies were higher among patients group $(92.8 \%)$ compared to $35 \%$ of their relatives. The presence of these antibodies in a control group was less temperature dependent, since $62.5 \%$ of them reacted both at $22 \mathrm{C}^{\circ}$ and $15 \mathrm{C}^{\circ}$ and only $12.5 \%$ of them exhibited a sole reactivity of $15 \mathrm{C}^{\circ}$.

No association between LCA and and of HLA antigen of both classes was detected, however, patients had a tendency to react with a large number of lymphocyte samples than did positive sera from other group. Generally, females had a higher percentage of LCA $(42.15 \%)$. Yet, the highest percentage was reported among sisters, followed by mothers, while brothers exhibited the least frequency (15.15\%). In contrast to females, male subjects reported the highest frequency of these antibodies among age group of $>50$ years.

Conclusion Patients expressed a high positivity rate of cold LCA. Moreover, female first degree relatives showed higher positivity rates than male relatives or controls.

\section{AB0072 LYMPHOCYTOTOXIC ANTIBODY (LCA) AMONG IRAQI SLE PATIENTS AND THEIR RELATIVES}

${ }^{1}$ AA Al-Shaikly, ${ }^{2} \mathrm{MM}$ Hasan, ${ }^{1} \mathrm{KM}$ Mousawy, ${ }^{1} \mathrm{~S}$ Salman. ${ }^{1}$ Faculty of Medicine; ${ }^{2}$ Faculty of Pharmacy, Baghdad University, Baghdad, Iraq

\subsection{6/annrheumdis-2001.117}

\section{Background}

Objectives To study the lymphocytotoxic antibodies (LCA) in SLE patients and their first degree relatives compared to matching controls.

Methods Complement dependent microcytotoxicity test was used to asses the presence of LCA, their temperature dependence in addition to HLA specificity among 40 SLE patients, 184 of their first degree relatives in comparison to 100 subjects of a blood bank donor as a healthy control.

Results Lyphocytotoxic activity was manifested in 70\% of patients, $33.36 \%$ of relatives compared to $80 \%$ of control group. While cold dependent antibodies were higher among patients group $(92.8 \%)$ compared to $35 \%$ of their relatives. The presence of these antibodies in a control group was less temperature dependent, since $62.5 \%$ of them reacted both at $22 \mathrm{C}^{\circ}$ and $15 \mathrm{C}^{\circ}$ and only $12.5 \%$ of them exhibited a sole reactivity of $15 \mathrm{C}^{\circ}$.

No association between LCA and any of HLA antigen of both classes was detected, however, patients had a tendency to react with a large number of lymphocyte samples than did positive sera from other group. Generally, females, had a higher percentage of Lca $(42.15 \%)$, yet, the highest percentage was reported among sisters, followed by mothers, while brothers exhibited the least frequency (15.15\%). In contrast to females, male subjects reported the highest frequency of these antibodies among age group of $>50$ years.

Conclusion Patients expressed a high positivity rate of cold LCA. Moreover, female first degree relatives showed higher positivity rates than male relatives or controls.

\section{AB0073 A CASE OF FULMINANT LUPUS IN A YOUNG MAN WITH HEREDITARY HAEMOCHROMATOSIS}

${ }^{1} \mathrm{CF}$ Matthews, ${ }^{2} \mathrm{P}$ Gardiner. ${ }^{1}$ Rheumatology, Musgrave Park Hospital, Belfast; ${ }^{2}$ Medicine, Altnagelvin Hospital, Londonderry, UK

\subsection{6/annrheumdis-2001.118}

Background Mild derangements of liver function tests in systemic lupus erythematosis (SLE) are not uncommon, reflecting either disease activity or treatment. Rare causes of hepatic dysfunction include arteritis and lupoid hepatitis. ${ }^{1}$ We report on a patient with SLE and coexisting haemochromatosis.

\section{Objectives}

Methods CASE REPORT: A 26 year old man presented with acute onset of jaundice and skin rash. He had a history of intermittent rash and arthralgia with serology positive for ribonuclear protein antibodies characteristic of mixed connective tissue disease. On examination he was icteric with a pyrexia. There was a maculopapular rash typical of subacute cutaneous lupus over his anterior chest wall.

Results Investigations showed a normocytic, normochromic anaemia, a thrombocytopaenia and a lymphopaenia. His prothrombin time and partial thromoboplastin time were prolonged. Liver function tests showed an elevated total bilirubin and raised aspartate transferase and alanine transferase. Autoimmune serology showed positive antiRNP/Sm. Complement levels were low. Anticardiolipin antibodies were negative. Viral titres were negative. Serum ferritin level was elevated. Blood cultures grew b haemolytic streptococcus. He was treated with high dose corticosteroids. He had intravenous antibiotics. The coagulopathy was treated with vitamin $\mathrm{K}$ and fresh frozen plasma. The thrombocytopaenia was treated with immunoglobulins. In spite of further management, unfortunately he died. A postmortem showed acute renal tubular necrosis and crescentic glomerulonephritis. Liver histology showed periportal deposition of iron predominately in zone three. This, together with the presence of iron deposition in the exocrine pancreas gland confirmed the diagnosis of hereditary haemochromatosis. 